\title{
Some Numerical Results on Fekete Polynomials
}

\author{
By Paul T. Bateman, George B. Purdy and Samuel S. Wagstaff, Jr.
}

Dedicated to Derrick H. Lehmer on the occasion of his seventieth birthday

\begin{abstract}
It is known that if $\chi$ is a real residue character modulo $k$ with $\chi(p)=-1$ for the first five primes $p$, then the corresponding Fekete polynomial $\Sigma_{n=1}^{k} \chi(n) x^{n}$ changes sign on $(0,1)$. In this paper it is shown that the condition that $\chi(p)$ be -1 for the first four primes $p$ is not sufficient to guarantee such a sign change. More specifically, if $\chi$ is the real nonprincipal character modulo either 1277 or 1973 , it is shown that the corresponding Fekete polynomial is positive throughout $(0,1)$ even though $\chi(2)=\chi(3)=\chi(5)=\chi(7)=-1$.
\end{abstract}

1. Introduction. Let $\chi$ be a real nonprincipal residue character to the modulus $k$. In this paper we consider the corresponding Fekete polynomial

$$
f(x, \chi)=\sum_{n=1}^{k} \chi(n) x^{n}
$$

and the related infinite series

$$
F(x, \chi)=\sum_{n=1}^{\infty} \chi(n) x^{n}=\left(1-x^{k}\right)^{-1} f(x, \chi) \quad(|x|<1) .
$$

Let $L(s, \chi)$ be the Dirichlet $L$-function defined by

$$
L(s, \chi)=\sum_{n=1}^{\infty} \chi(n) n^{-s} \quad(\operatorname{Re} s>0)
$$

In view of the relation

$$
\begin{aligned}
\Gamma(s) L(s, \chi) & =\int_{0}^{\infty} u^{s-1} F\left(e^{-u}, \chi\right) d u \\
& =\int_{0}^{\infty} u^{s-1}\left(1-e^{-k u}\right)^{-1} f\left(e^{-u}, \chi\right) d u \quad(\operatorname{Re} s>0),
\end{aligned}
$$

it is immediate that if $f(x, \chi)$ or $F(x, \chi)$ is positive for all $x$ in $(0,1)$, then $L(s, \chi)$ is positive for all positive $s$.

Unfortunately, it very often happens that $f(x, \chi)$ changes sign in $(0,1)$. The purpose of this paper is to shed a little light on the extent to which this is true.

Heilbronn [5] proved that there is a prime number $P$ such that if $\chi(p)=-1$ for all primes $p$ not exceeding $P$, then $f(x, \chi)$ takes some negative values on $(0,1)$.

Received July 22, 1974.

AMS (MOS) subject classifications (1970). Primary 10-04, 10H10, 10H20; Secondary 26A78. Key words and phrases. Real residue characters, Chowla's method, Dirichlet $L$-functions, Fekete polynomials, real zeros of polynomials. 
It may be of some interest to determine the smallest value of $P$ for which Heilbronn's assertion is correct.

On the one hand we cannot take $P=5$. For if $\chi(n)=(n / 53)$, then $f(x, \chi)$ is positive throughout $(0,1)$, since in this case $(1-x)^{-3} f(x, \chi)$ has a power series around the origin with all coefficients positive (cf. [9]).

On the other hand we have the following three quantitative forms of Heilbronn's theorem:

I. (Cf. p. 37 of [7] or Problem 46 of Part V of [8].) If $\chi(p)=-1$ for all primes $p$ not exceeding 11 , then

$$
\begin{aligned}
f(x, \chi) \leqslant & x-x^{2}-x^{3}+x^{4}-x^{5}+x^{6}-x^{7}-x^{8}+x^{9}+x^{10}-x^{11}-x^{12} \\
& +\sum_{n=13}^{\infty} x^{n}
\end{aligned}
$$

and the right-hand side is negative when $x=0.7$ (actually throughout the closed interval $[0.69,0.80])$.

II. (Cf. p. 31 of [3].) If $\chi(p)=-1$ for all primes $p$ not exceeding 7 and if in addition $\chi(p)=-1$ for at least one of the primes $p=11,13,17,19$, then

$$
\begin{aligned}
f(x, \chi) \leqslant & x-x^{2}-x^{3}+x^{4}-x^{5}+x^{6}-x^{7}-x^{8}+x^{9}+x^{10}+x^{11}-x^{12} \\
& +x^{13}+x^{14}+x^{15}+x^{16}+x^{17}-x^{18}-x^{19}-x^{20}+\sum_{n=21}^{\infty} x^{n}
\end{aligned}
$$

and the right-hand side is negative when $x=0.74$ (actually throughout the closed interval $[0.738,0.749])$.

III. (Cf. [4].) If $\chi(p)=-1$ for all primes $p$ not exceeding 7 and if in addition $\chi(p)<1$ for at least one of the primes $p=11,13,17,19,23$, then $f(0.745, \chi)<0$.

Since Hahn's result III is a little more complicated than I or II, we give a detailed proof in Section 2 for completeness.

The results I, II, and III show that we can take $P=11$ in Heilbronn's theorem and hint that perhaps it might even be possible to take $P=7$. However, in this paper we shall show, by considering the real nonprincipal characters with moduli 1277 and 1973, that we cannot take $P=7$ in Heilbronn's theorem. Specifically we obtain the following numerical result.

THEOREM. There are 141 real primitive characters $\chi$ with modulus less than 10080 for which $\chi(2)=\chi(3)=\chi(5)=\chi(7)=-1$. Of these 141 characters (a) 138 satisfy the hypotheses of assertion III and so the corresponding Fekete polynomials change sign in $(0,1),(b)$ the character $\chi(n)=(n / 9907)$ does not satisfy the hypotheses of III but nevertheless the corresponding Fekete polynomial does change sign in $(0,1),(c)$ the Fekete polynomials corresponding to the two characters (n/1277), $(n / 1973)$ are positive throughout $(0,1)$. 
COROLlARY. The Dirichlet L-functions formed from the two characters mentioned in (c) and the Dedekind zeta functions for the quadratic fields with discriminants 1277 and 1973 have no positive real zeros.

We remark that there are 135 imprimitive real characters $\chi$ with moduli less than 10080 for which $\chi(2)=\chi(3)=\chi(5)=\chi(7)=-1$. These characters are obtained by multiplying the real primitive characters with moduli $43,67,163,173$, $293,403,437,547,667,677,773,797,883,907$ by various principal characters. These 135 imprimitive real characters all satisfy the hypotheses of assertion III and so the corresponding Fekete polynomials all change sign in $(0,1)$.

Two different methods of proof are used for part (c) of the theorem. One method is based on a direct calculation of the values of the polynomial $g(x, \chi)=x^{-1} f(x, \chi)$ and the use of the bound $(1-x)^{-2}$ for its derivative. In addition we require an arithmetical lemma showing that $f(x, \chi)$ is positive on a small open interval with 1 as its right-hand endpoint.

The other method is based on finding a positive integer $m$ such that the power series for $(1-x)^{-m} F(x, \chi)$ has all coefficients positive. In the case $\chi(n)=(n / 1973)$, we find that $m=567$ is the smallest value of $m$ which will work. In the case $\chi(n)$ $=(n / 1277)$, we find that $m=766$ is the smallest value of $m$ which will work. This second method is often attributed to Chowla [1], but in fact goes back to Fekete (cf. [2]). While the Fekete-Chowla method is sometimes useful for characters of small moduli, in the cases at hand it takes over 100 times as much computer time as the first method.

It should be emphasized that the positivity of the Fekete polynomial $f(x, \chi)$ throughout $(0,1)$ is merely a crude sufficient condition for the positivity of $L(s, \chi)$ for all positive $s$. When $\chi(-1)=-1$ a much more subtle way of obtaining the positivity of $L(s, \chi)$ for all positive $s$ is presented in [6] and in a forthcoming paper by Purdy.

2. Proof of III. Let $\lambda$ denote the Liouville function and let $*$ indicate summation over those values of $n$ having no prime factors greater than 7 . Then under the hypotheses of III we have

$$
\begin{aligned}
f(x, \chi)< & \sum_{n=1}^{39} \chi(n) x^{n}+\sum_{n=40}^{\infty} x^{n} \\
< & \sum_{n<40}^{*} \lambda(n) x^{n}+x^{29}+x^{31}+x^{37}+x^{40} /(1-x) \\
& +\chi(11)\left\{x^{11}-x^{22}-x^{33}\right\}+\chi(13)\left\{x^{13}-x^{26}-x^{39}\right\} \\
& +\chi(17)\left\{x^{17}-x^{34}\right\}+\chi(19)\left\{x^{19}-x^{38}\right\}+\chi(23) x^{23} .
\end{aligned}
$$

For $0<x<0.8$ we have

$$
x^{11}-x^{22}-x^{33}>x^{13}-x^{26}-x^{39}>x^{17}-x^{34}>x^{19}-x^{38}>x^{23} .
$$


In view of these inequalities the extremal case of the above majorant for $f(x, \chi)$ consistent with the hypotheses of III is that in which

$$
\chi(11)=\chi(13)=\chi(17)=\chi(19)=1, \quad \chi(23)=0 .
$$

Thus for $0<x<0.8$ we have

$$
f(x, \chi)<\sum_{n=1}^{39} \rho(n) x^{n}+x^{40} /(1-x)=P(x),
$$

where $\rho$ is a completely multiplicative arithmetic function such that

$$
\begin{gathered}
\rho(2)=\rho(3)=\rho(5)=\rho(7)=-1, \quad \rho(11)=\rho(13)=\rho(17)=\rho(19)=1, \\
\rho(23)=0, \quad \rho(29)=\rho(31)=\rho(37)=1 .
\end{gathered}
$$

The rational function $P(x)$ is negative when $x=0.745$ and in fact throughout the entire closed interval $[0.742,0.749]$. However, the single calculation $P(0.745)<$ -0.0001488 is all that is logically required. Thus III is proved.

We remark that if $\chi(2)=\chi(3)=\chi(5)=\chi(7)=-1$ but $\chi(11)=\chi(13)=$ $\chi(17)=\chi(19)=\chi(23)=1$, then

$$
\begin{aligned}
f(x, \chi)> & x-x^{2}-x^{3}+x^{4}-x^{5}+x^{6}-x^{7}-x^{8}+x^{9}+x^{10}+x^{11}-x^{12} \\
& +x^{13}+x^{14}+x^{15}+x^{16}+x^{17}-x^{18}+x^{19}-x^{20}+x^{21}-x^{22} \\
& +x^{23}+x^{24}+x^{25}-\sum_{n=26}^{\infty} x^{n}
\end{aligned}
$$

which can be shown to be positive on the interval $(0,0.9217)$ by the method of Section 5. Thus the condition in III that at least one of the numbers $\chi(11), \chi(13), \chi(17)$, $\chi(19), \chi(23)$ be less than one is absolutely essential. Of course this is shown more forcefully by part (c) of the theorem.

3. Proof of Part (a) of the Theorem. Consider a real primitive character $\chi$ with $\chi(2)=\chi(3)=\chi(5)=\chi(7)=-1$. Since $\chi(2) \neq 0$, the modulus of $\chi$ must be odd. Thus $\chi$ is given by the Jacobi symbol

$$
\chi(n)=(n / q)
$$

for some odd squarefree positive integer $q$ (not necessarily a prime). By using the quadratic reciprocity law and its two supplements we find that

$$
\begin{gathered}
q \equiv 3,5 \quad(\bmod 8), \quad q \equiv 5,7 \quad(\bmod 12), \quad q \equiv 2,3 \quad(\bmod 5) \\
q \equiv 5,11,13,15,17,23 \quad(\bmod 28)
\end{gathered}
$$

Thus

$$
q \equiv 43,67,163,173,293,403,437,547,667,677,773,797 \quad(\bmod 840) .
$$


When we confine ourselves to values of $q$ less than $10080=12 \cdot 840$, we obtain twelve possible $q$ in each of the twelve listed residue classes modulo 840 . However the three numbers

$$
5203=43 \cdot 11^{2}, \quad 7267=43 \cdot 13^{2}, \quad 8107=67 \cdot 11^{2}
$$

are not squarefree and therefore do not give rise to primitive characters.

By considering $\chi(11), \chi(13), \chi(17), \chi(19)$, and $\chi(23)$ we find that the remaining 141 values of $q$ fall into four classifications according to whether or not the hypotheses of I, II, and III are satisfied. For the following 66 values of $q$ the corresponding character $\chi$ given by $(*)$ satisfies the hypotheses of $\mathrm{I}$ :

$67,163,173,293,437,677,883,907,1387,1517,1613,1747,2083,2227,2347$, $2477,2813,3067,3197,3317,3403,3523,3533,3763,3797,4027,4133,4157$, $4373,4603,4637,4867,5083,5107,5213,5443,5477,5707,5717,5837,5923$, $6043,6173,6427,6763,6893,7013,7157,7493,7627,7723,7853,8333,8357$, 8563, 8803, 8947, 9067, 9077, 9173, 9307, 9403, 9413, 9533, 9677, 9917.

For the following 65 values of $q$ the corresponding character $\chi$ given by $(*)$ satisfies the hypotheses of II but not the hypotheses of I:

$43,403,547,773,797,1003,1013,1133,1243,1507,1637,1723,1843,1853$, $2117,2357,2453,2563,2587,2683,2693,2923,2957,3187,3293,3427,3907$, 4037, 4243, 4267, 4363, 4493, 4747, 4877, 4973, 4997, 5333, 5587, 5813, 5947, $6053,6283,6317,6547,6557,6653,6677,6787,6883,7123,7517,7603,7733$, 7963, 7997, 8227, 8237, 8443, 8573, 8693, 8837, 9197, 9283, 9787, 10037.

For the following 7 values of $q$ the corresponding character $\chi$ given by $(*)$ satisfies the hypotheses of III but not the hypotheses of II:

$$
667,3653,7387,7397,8467,9643,10013 .
$$

Finally for the following 3 values of $q$ the corresponding character $\chi$ given by $(*)$ does not satisfy the hypotheses of III: 1277, 1973, 9907. A table of some of the values of the ten primitive characters falling into the last two classifications is given in Table 1.

Altogether there are $66+65+7=138$ values of $q$ such that the corresponding primitive character $\chi$ satisfies the hypotheses of III, and accordingly $f(x, \chi)<0$ when $x=0.745$ in all these cases.

4. Proof of Part (b) of the Theorem. While the character $\chi(n)=(n / 9907)$ is not covered by III, a straightforward calculation and a rough error analysis reveals that in this case $f(0.983, \chi)<-0.37$. We shall indicate in Section 5 how we arrived at the value 0.983 . However, the relatively large absolute value of $f(0.983, \chi)$ indicates that the calculation required here is not a very delicate one. For example, if we merely wished to prove the negativity of $f(0.983, \chi)$, we could take only the first 400 terms 
TABLE 1

Real primitive characters $\chi$ with $\chi(2)=\chi(3)=\chi(5)=\chi(7)=-1$ and $\chi(11)>-1, \chi(13)>-1, \chi(17)>-1, \chi(19)>-1$.

$n\left(\frac{n}{667}\right)\left(\frac{n}{1277}\right)\left(\frac{n}{1973}\right)\left(\frac{n}{3653}\right)\left(\frac{n}{7387}\right)\left(\frac{n}{7397}\right)\left(\frac{n}{8467}\right)\left(\frac{n}{9643}\right)\left(\frac{n}{9907}\right)\left(\frac{n}{10013}\right)$

\begin{tabular}{lllllllllll}
\hline 11 & + & + & + & + & + & + & + & + & + & + \\
13 & + & + & + & 0 & + & 0 & + & + & + & + \\
17 & + & + & + & + & + & + & + & + & + & 0 \\
19 & + & + & + & + & + & + & + & + & + & 0 \\
23 & 0 & + & + & - & - & - & - & - & + & + \\
29 & 0 & + & + & + & - & - & + & - & - & - \\
31 & - & - & + & - & - & + & - & - & - & 0 \\
37 & + & - & + & + & - & + & - & - & + & - \\
41 & - & - & + & + & - & - & + & + & - & + \\
43 & + & - & + & + & + & + & + & - & - & - \\
47 & - & + & - & + & - & + & - & - & - & + \\
53 & - & - & - & + & - & - & + & - & + & + \\
59 & + & - & + & - & - & + & + & - & + & - \\
61 & + & + & - & - & - & + & + & + & + & + \\
67 & - & + & - & + & - & - & - & - & + & - \\
71 & + & - & - & + & - & - & - & - & - & + \\
73 & - & + & + & + & - & + & + & - & - & + \\
79 & + & + & - & + & - & + & + & - & - & - \\
83 & - & - & + & + & 0 & + & - & + & - & - \\
89 & + & - & - & + & 0 & + & - & - & - & + \\
\hline
\end{tabular}

of $f(x, \chi)$, say, since

$$
\sum_{n=401}^{\infty}(0.983)^{n}=\frac{983}{17}(0.983)^{400}<0.061 .
$$

This shows that $f(0.983, \chi)<-0.3$ for any $\chi$ of the form

$$
\chi(n)=(n / q), \quad q \equiv 9907 \quad(\bmod 4 M),
$$

where $M$ is the product of all primes less than 400 .

5. Proof of Part (c) of the Theorem by Direct Calculation. We require some lemmas.

LEMma 1 (Cf. p. 17 of [3]). Suppose $\chi$ is a primitive residue character modulo $k$ with $k>1$ and $\chi(-1)=1$. Let $f(x)=f(x, \chi)$ and $\rho=e^{2 \pi i / k}$. Then for $|z|<1$ we have

$$
\frac{f\left(e^{-2 \pi z / k}\right)}{1-e^{-2 \pi z}}=\frac{f(\rho)}{\pi} \sum_{n=0}^{\infty}(-1)^{n} z^{2 n+1} L(2 n+2, \bar{\chi}) .
$$

Proof. Since $f\left(\rho^{m}\right)=\bar{\chi}(m) f(\rho)$ for any positive integer $m$, we have 


$$
\frac{f(x)}{1-x^{k}}=\sum_{m=1}^{k} \frac{f\left(\rho^{m}\right)}{k} \frac{1}{1-\rho^{-m} x}=\sum_{m=1}^{k} \frac{f(\rho)}{k} \frac{\bar{\chi}(m)}{1-\rho^{-m} x} .
$$

Thus for $|z|<1$ we have

$$
\begin{aligned}
\frac{f\left(e^{-2 \pi i z / k}\right)}{1-e^{-2 \pi i z}} & =\frac{f(\rho)}{k} \quad \sum_{m=1}^{k} \bar{\chi}(m)\left\{\frac{1}{\left.1-e^{-2 \pi i(z+m) / k}-\frac{1}{2}\right\}}\right. \\
& =\frac{f(\rho)}{2 k i} \sum_{m=1}^{k} \bar{\chi}(m) \operatorname{cotg} \frac{\pi}{k}(z+m) \\
& =\frac{f(\rho)}{2 k i} \sum_{m=1}^{k} \bar{\chi}(m) \lim _{J \rightarrow+\infty} \sum_{j=-J}^{J} \frac{1}{\pi(z+m) / k-\pi j} \\
& =\frac{f(\rho)}{2 \pi i} \lim _{J \rightarrow+\infty} \sum_{j=-J}^{J} \sum_{m=1}^{k} \frac{\bar{\chi}(m-k j)}{z+m-k j} \\
& =\frac{f(\rho)}{2 \pi i} \lim _{M \rightarrow+\infty} \sum_{m=-M}^{M} \frac{\bar{\chi}(m)}{z+m} \\
& =\frac{f(\rho)}{2 \pi i} \lim _{M \rightarrow+\infty} \quad \sum_{0<|m| \leqslant M} \frac{\bar{\chi}(m)}{m} \sum_{r=0}^{\infty}\left(-\frac{z}{m}\right)^{r} \\
& =\frac{f(\rho)}{2 \pi i} \sum_{r=0}^{\infty}(-z)^{r} \lim _{M \rightarrow+\infty} \quad \sum_{0<|m| \leqslant M} \bar{\chi}(m) m^{-r-1},
\end{aligned}
$$

since if we separate out the terms with $r=0$, the remaining iterated sum is absolutely convergent. The result of the lemma follows upon replacing $z$ by $-i z$ and using the assumption $\chi(-1)=1$.

LEMMA 2. If $\chi$ is a primitive real character modulo $k$ with $k>1$ and $\chi(-1)=1$, then $f(x, \chi)>0$ when $\exp (-2 \sqrt{7} / k)<x<1$.

Proof. Recall that for a real primitive character $\chi$ modulo $k$ we have

$$
f\left(e^{2 \pi i / k}, \chi\right)=\sum_{m=1}^{k} \chi(m) e^{2 \pi i m / k}=\{\chi(-1) k\}^{1 / 2} .
$$

Thus if $|z|<1$, we have by Lemma 1

$$
f\left(e^{-2 \pi z / k}, \chi\right)=z\left(1-e^{-2 \pi z}\right) \pi^{-1} k^{1 / 2} \sum_{n=0}^{\infty}(-1)^{n} z^{2 n} L(2 n+2, \chi) .
$$

Now if $n$ is a positive integer,

$$
\begin{aligned}
\frac{L(2 n+2, \chi)}{L(2 n, \chi)} & =\prod_{p} \frac{1-\chi(p) p^{-2 n}}{1-\chi(p) p^{-2 n-2}} \leqslant \prod_{\chi(p)=-1} \frac{1+p^{-2 n}}{1+p^{-2 n-2}} \\
& \leqslant \prod_{p} \frac{1+p^{-2}}{1+p^{-4}}=\frac{\zeta(2) \zeta(8)}{\zeta(4)^{2}}=\frac{\pi^{2}}{7} .
\end{aligned}
$$


Thus if $0<z<\sqrt{7} / \pi$, the $n$th term of the above series for $f\left(e^{-2 \pi z / k}, \chi\right)$ decreases in absolute value as $n$ increases. Hence $f\left(e^{-2 \pi z / k}, \chi\right)>0$ if $0<z<\sqrt{7} / \pi$ and so $f(x, \chi)>0$ if $\exp (-2 \sqrt{7} / k)<x<1$.

For later reference we note that

$$
\exp (-2 \sqrt{7} / 1277)<0.9960, \quad \exp (-2 \sqrt{7} / 1973)<0.9975 .
$$

LeMma 3. For a real residue character $\chi$ let

$$
g(x)=g(x, \chi)=x^{-1} f(x, \chi)=\sum_{n=1}^{k} \chi(n) x^{n-1} .
$$

If $g\left(x_{0}, \chi\right)>0$ for some $x_{0}$ in $[0,1)$, then $g(x, \chi)>0$ on the interval $\left(x_{0}, x_{0}+\right.$ $\left.\delta\left(x_{0}\right)\right)$, where

$$
\delta\left(x_{0}\right)=g\left(x_{0}\right)\left(1-x_{0}\right)^{2} /\left(1+g\left(x_{0}\right)\left(1-x_{0}\right)\right) .
$$

Proof. We remark that

$$
g\left(x_{0}\right)=\sum_{n=1}^{k} \chi(n) x_{0}^{n-1}<\sum_{n=1}^{\infty} x_{0}^{n-1}=\left(1-x_{0}\right)^{-1},
$$

so that $\delta\left(x_{0}\right)<1-x_{0}$ and $x_{0}+\delta\left(x_{0}\right)$ is still in $[0,1)$. Now

$$
g^{\prime}(x, \chi)=\sum_{n=2}^{k} \chi(n)(n-1) x^{n-2}>-\sum_{n=2}^{\infty}(n-1) x^{n-2}=-(1-x)^{-2} \text {. }
$$

Thus if $0<h<\delta\left(x_{0}\right)$, we have

$$
\begin{aligned}
g\left(x_{0}+h\right) & =g\left(x_{0}\right)+\int_{x_{0}}^{x_{0}+h} g^{\prime}(t) d t \geqslant g\left(x_{0}\right)-\int_{x_{0}}^{x_{0}+h}(1-t)^{-2} d t \\
& =g\left(x_{0}\right)-\frac{h}{\left(1-x_{0}\right)\left(1-x_{0}-h\right)} \\
& =\frac{g\left(x_{0}\right)\left(1-x_{0}\right)^{2}-h\left\{1+g\left(x_{0}\right)\left(1-x_{0}\right)\right\}}{\left(1-x_{0}\right)\left(1-x_{0}-h\right)}>0 .
\end{aligned}
$$

LEMMA 4. If $\chi$ is a real residue character and if $g\left(x_{0}, \chi\right)=x^{-1} f\left(x_{0}, \chi\right)>0$ for some $x_{0}$ in $[1 / 2,1)$, then $g(x, \chi)>0$ on the interval $\left(x_{0}-\eta\left(x_{0}\right), x_{0}\right)$, where

$$
\eta\left(x_{0}\right)=g\left(x_{0}\right)\left(1-x_{0}\right)^{2}>\delta\left(x_{0}\right) .
$$

Proof. We remark that $\eta\left(x_{0}\right)<1-x_{0} \leqslant x_{0}$, so that $x_{0}-\eta\left(x_{0}\right)$ is still in $[0,1)$. If $0<h<\eta\left(x_{0}\right)$, we have

$$
g\left(x_{0}-h\right)=g\left(x_{0}\right)-\int_{x_{0}-h}^{x_{0}} g^{\prime}(t) d t \geqslant g\left(x_{0}\right)-h\left(1-x_{0}\right)^{-2}>0 .
$$

Now we apply these lemmas and some machine computation to prove part (c) of our theorem. In view of Lemma 2 it suffices to show that 
(*)

and

$$
g(x, \chi)>0 \text { for } 0 \leqslant x \leqslant 0.9960, \text { when } \chi(n)=(n / 1277)
$$

$(* *) \quad g(x, \chi)>0$ for $0 \leqslant x \leqslant 0.9975$, when $\chi(n)=(n / 1973)$.

To prove $(*)$ and $(* *)$ we used the computer to construct a finite sequence of numbers $x_{1}, x_{2}, \cdots, x_{N}$ such that

(i) $x_{1}=0$,

(ii) $x_{j+1}=x_{j}+\delta\left(x_{j}\right)$,

(iii) $g\left(x_{j}\right)>0$,

(iv) $x_{N}>0.9960$ when $\chi(n)=(n / 1277), x_{N}>0.9975$ when $\chi(n)=(n /$ i 973$)$.

The existence of such a sequence is a necessary and sufficient condition for $(*)$ or $(* *)$, respectively.

For the real nonprincipal character modulo 1973 we found $N=696$. The smallest value of $g\left(x_{j}\right)=g\left(x_{j}, \chi\right)$ found was

$$
g\left(x_{157}, \chi\right)=g(0.7440438789)=0.0012990974,
$$

while the smallest value of $\delta\left(x_{j}\right)$ used was

$$
\delta\left(x_{695}\right)=\delta(0.9974918304)=0.0000243189 .
$$

Fortunately $\delta$ was often much larger than this minimum value, so that the length of our sequence remained within reasonable bounds. A skeleton table of values, including the relative extrema of the sequence $g\left(x_{j}\right)$, is given in Table 2 .

For the real nonprincipal character modulo 1277 we found $N=864$. The smallest value of $g\left(x_{j}\right)$ found was

$$
g\left(x_{188}, \chi\right)=g(0.7445806130)=0.0009461806
$$

and the smallest value of $\delta\left(x_{j}\right)$ found was

$$
\delta\left(x_{613}\right)=\delta(0.9817924724)=0.0000268077 .
$$

Table 3 gives a skeleton table of values, including the relative extrema of the sequence $g\left(x_{j}\right)$.

For both cases Lemma 3 shows that $f(x, \chi)>0$ on each interval $\left(x_{j}, x_{j+1}\right)$ for $j=1,2, \cdots, N-1$. Thus $f(x, \chi)>0$ throughout $(0,1)$ for the nonprincipal characters with moduli 1277 and 1973.

While we have not used Lemma 4 as such, it shows that once we obtain a positive value for $g\left(x_{j+1}, \chi\right)$ we have a second argument for showing the positivity of $g(x, \chi)$ for at least the right-hand part of $\left(x_{j}, x_{j+1}\right)$. It could also be used to produce an alternative proof of part (c) of the theorem by constructing a sequence going in the opposite direction, starting from 0.9960 or 0.9975 .

We remark that in a case where $g(x, \chi)=x^{-1} f(x, \chi)$ has a zero in $[0,1)$ the sequence constructed by using (i), (ii), and (iii) indefinitely will be infinite and will 
TABLE 2

Some values of $g(x, \chi)=\Sigma_{n=1}^{1972} \chi(n) x^{n-1}$, where $\chi(n)=(n / 1973)$

\begin{tabular}{rlcc}
\hline$j$ & \multicolumn{1}{c}{$x_{j}$} & \multicolumn{1}{c}{$g\left(x_{j}\right)$} & $\delta\left(x_{j}\right)$ \\
\hline 1 & 0.0 & 1.0000000000 & 0.5000000000 \\
\hline 3 & 0.5000000000 & 0.3271283007 & 0.0702858327 \\
156 & 0.5702858327 & 0.2121684340 & 0.0359043434 \\
157 & 0.7439587333 & 0.0012992317 & 0.0000851456 \\
158 & 0.7440438789 & 0.0012990974 & 0.0000850801 \\
347 & 0.7441289590 & 0.0012991815 & 0.0000850292 \\
348 & 0.9483029621 & 1.6113573903 & 0.0039753328 \\
349 & 0.9522782949 & 1.6293312786 & 0.0034428767 \\
463 & 0.9557211716 & 1.6267671765 & 0.0029751588 \\
464 & 0.9880324013 & 0.2587139465 & 0.0000369396 \\
465 & 0.9880693409 & 0.2586946663 & 0.0000367094 \\
695 & 0.9881060503 & 0.2587203522 & 0.0000364879 \\
696 & 0.9974918304 & 3.9035744175 & 0.0000243189 \\
697 & 0.9975161493 & 3.9075094417 & 0.0000238757 \\
701 & 0.9975400250 & 3.9107595096 & 0.0000234404 \\
702 & 0.9976312533 & 3.9173067427 & 0.0000217778 \\
703 & 0.9976530311 & 3.9174293158 & 0.0000213817 \\
& 0.9976744128 & 3.9169854994 & 0.0000209932 \\
& & &
\end{tabular}

converge to the smallest positive zero of $g(x, \chi)$. In fact, in the case of the real nonprincipal character modulo 9907 the present method determined that the first positive zero of $g(x, \chi)$ is about 0.971780446 . The function $g(x, \chi)$ becomes negative after this value and reaches a relative minimum of about -0.3775 when $x$ is about 0.983 . This explains how we chose the value 0.983 as a suitable point at which to evaluate $f(x, \chi)=x g(x, \chi)$ for the modulus 9907.

Needless to say it is not really necessary to begin our sequences $x_{1}, x_{2}, \cdots$ with $x_{1}=0$. For example, it is easy to prove (cf. pp. 28-29 of [3]) that for an arbitrary real character $\chi$ we have $f(x, \chi)>0$ on the interval $(0, \xi]$, where $\xi=0.6707 \cdots$ is the smallest positive zero of the power series $\Sigma_{n=1}^{\infty} \lambda(n) x^{n}$. Thus we could begin our sequence with $x_{1}=0.67$, say. In a similar way we could begin our sequences with $x_{1}=0.92$ by using the result quoted at the end of Section 2 , namely that for characters satisfying

$$
\chi(2)=\chi(3)=\chi(5)=\chi(7)=-1, \quad \chi(11)=\chi(13)=\chi(17)=\chi(19)=\chi(23)=1
$$

we have

$$
g(x, \chi)=x^{-1} f(x, \chi)>0 \text { for } 0<x<0.9217
$$


TABLE 3

Some values of $g(x, \chi)=\Sigma_{n=1}^{1276} \chi(n) x^{n-1}$, where $\chi(n)=(n / 1277)$.

\begin{tabular}{rlcl}
\hline$j$ & $x_{j}$ & $g\left(x_{j}\right)$ & $\delta\left(x_{j}\right)$ \\
\hline 1 & 0.0 & 1.0000000000 & 0.5000000000 \\
3 & 0.5000000000 & 0.3271282988 & 0.0702858323 \\
187 & 0.5702858323 & 0.2121683345 & 0.0359043280 \\
188 & 0.7445188700 & 0.0009461824 & 0.0000617430 \\
189 & 0.7445806130 & 0.0009461806 & 0.0000617130 \\
409 & 0.7446423260 & 0.0009462923 & 0.0000616905 \\
410 & 0.9306691788 & 0.9233622379 & 0.0041713443 \\
411 & 0.9348405231 & 0.9286600710 & 0.0037178921 \\
582 & 0.9385584152 & 0.9242526263 & 0.0033016256 \\
583 & 0.9809481090 & 0.0774233276 & 0.0000280613 \\
584 & 0.9809761703 & 0.0774219891 & 0.0000279783 \\
863 & 0.9810041486 & 0.0774283493 & 0.0000278984 \\
864 & 0.9959763690 & 3.0991683047 & 0.0000495564 \\
865 & 0.9960259254 & 3.1060949352 & 0.0000484572 \\
871 & 0.9960743826 & 3.1120902540 & 0.0000473800 \\
872 & 0.9963432772 & 3.1301413811 & 0.0000413814 \\
873 & 0.9963846586 & 3.1304408794 & 0.0000404592 \\
& 0.9964251178 & 3.1300456513 & 0.0000395586
\end{tabular}

However, the detailed proof of this latter result would require exactly the same method we have just been using.

A word must be said about roundoff error in calculating $g(x, \chi)$ for the real nonprincipal characters with moduli 1277,1973 , and 9907 . If the value of a polynomial $a_{0}+a_{1} x+\cdots+a_{m} x^{m}$ is calculated for a particular value of $x$ by the customary method of synthetic division on a machine whose arithmetic operations are rounded off to $t$ binary digits, the result obtained is

$$
a_{m}\left(1+E_{m}\right) x^{m}+a_{m-1}\left(1+E_{m-1}\right) x^{m-1}+\cdots+a_{1}\left(1+E_{1}\right) x+a_{0}\left(1+E_{0}\right),
$$

where

$$
\left(1-2^{-t}\right)^{2 r+2} \leqslant 1+E_{r} \leqslant\left(1+2^{-t}\right)^{2 r+2} \quad(r=0,1, \cdots, m) .
$$

(Cf. pp. 49-50 of [10].) For the moduli 1277 and 1973 we have $\left|a_{i}\right|=1$, $m<2000$, and $0<x<1$. For the IBM 360 (which was used) we have $t \geqslant 49$. Thus

$$
1+E_{r} \leqslant\left(1+2^{-49}\right)^{4000} \leqslant\left(e^{2^{-49}}\right)^{2^{12}}=e^{2^{-37}}<1+2^{-36.6}<1+10^{-11},
$$

and

$$
1+E_{r} \geqslant\left(1-2^{-49}\right)^{4000} \geqslant\left(e^{2^{-48.6}}\right)^{2^{12}}=e^{-2^{-36.6}}>1-2^{-36.6}>1-10^{-11}
$$


Thus

$$
\left|E_{r}\right|<10^{-11} \quad(r=0,1, \cdots, m) .
$$

Hence the roundoff error in calculating one of the values of $g(x, \chi)$ for the real nonprincipal characters with moduli 1277 and 1973 is less than $2(10)^{-8}$. Thus the first seven of the ten decimal places printed out for the values of $g(x, \chi)$ are significant in these two cases. Similarly the roundoff error in calculating $g(x, \chi)$ for the real nonprincipal character modulo 9907 is less than $10^{-6}$ and so our assertion that $f(0.983, \chi)<-0.37$ in that case is certainly correct.

For the moduli 1277 and 1973 we can say that, since each value of $g(x, \chi)$ is calculated from scratch and since the values obtained are all greater than 0.0009 , the correct functional values at the points chosen are certainly positive. The only question remaining is whether we can be sure of positivity in $\left(x_{j}, x_{j+1}\right)$. Once $x_{j}$ is chosen, we certainly know positivity in the interval $\left(x_{j}, x_{j}+\delta\left(x_{j}\right)\right)$. The only problem is that there may be a roundoff error in calculating

$$
\delta\left(x_{j}\right)=g\left(x_{j}\right)\left(1-x_{j}\right)^{2} /\left(1+\left(1-x_{j}\right) g\left(x_{j}\right)\right) .
$$

However, this roundoff error is certainly less than $10^{-6}$. But if we apply Lemma 4 to the computed value of $x_{j+1}$, we can infer positivity of $g(x, \chi)$ in an interval to the left of $x_{j+1}$ of length at least $\eta\left(x_{j+1}\right)>\delta\left(x_{j+1}\right)>0.00002>10^{-6}$. Thus the possible roundoff error in $\delta\left(x_{j}\right)$ does not raise any question about the positivity of $g(x, \chi)$ in $\left(x_{j}, x_{j+1}\right)$, since we have the reserve firepower of Lemma 4 .

6. Proof of Part (c) of the Theorem by the Fekete-Chowla Method. The following lemma is due to Fekete.

Lemma 5. If $f$ is a polynomial with real coefficients, then $f$ has no zeros in $(0,1)$ if and only if there is a nonnegative integer $m$ such that the power series for $(1-x)^{-m} f(x)$ around the origin has all coefficients of one sign.

Proof. (Cf. p. 99 of [2] and Problems 44 and 187 of Part V of [8].) The proof consists in noting that a real polynomial with no zeros in $(0,1)$ can be factored into real factors of the following types:

$$
\begin{array}{ll}
d x^{j}, & \text { where } d \neq 0 \text { and } j \text { is a nonnegative integer; } \\
1-h x, & \text { where } 0<h \leqslant 1 \\
1+k x, & \text { where } k>0 \\
a x^{2}+b x+c, & \text { where } a>0, c>0,4 a c>b^{2} .
\end{array}
$$

Now

$$
(1-x)^{-1}(1-h x)=1+(1-h) x+(1-h) x^{2}+\cdots
$$

and it can be verified that the power series for $(1-x)^{-r}\left(a x^{2}+b x+c\right)$ has positive coefficients if 


$$
r>\max \left(-\frac{b}{c}, \frac{a+b+c}{2(a c)^{1 / 2}+b}+1\right) .
$$

The result of the lemma then follows by multiplication.

If $\chi$ is a given real nonprincipal character modulo $k$ and if $m$ is a nonnegative integer, put

and

$$
\sum_{n=1}^{\infty} s_{m}(n) x^{n}=(1-x)^{-m} f(x, \chi)=(1-x)^{-m}\left(1-x^{k}\right) F(x, \chi)
$$

$$
\sum_{n=1}^{\infty} S_{m}(n) x^{n}=(1-x)^{-m} F(x, \chi)=(1-x)^{-m}\left(1-x^{k}\right)^{-1} f(x, \chi) .
$$

Thus $s_{0}(n)=\chi(n)$ if $1 \leqslant n \leqslant k, s_{0}(n)=0$ for $n>k$, and $S_{0}(n)=\chi(n)$ for all positive integral $n$. Also we have the recursion relations

$$
s_{m}(n)=\sum_{j=1}^{n} s_{m-1}(j), \quad S_{m}(n)=\sum_{j=1}^{n} S_{m-1}(j)
$$

valid for positive integral $m$ and $n$. With this notation we have the following two lemmas.

LEмма 6. If $\chi$ is a real nonprincipal character modulo $k$, the following four assertions are equivalent:

(i) $F(x, \chi)$ is positive throughout $(0,1)$,

(ii) $f(x, \chi)$ is positive throughout $(0,1)$,

(iii) there is a positive integer $m$ such that $s_{m}(n) \geqslant 0$ for all $n$,

(iv) there is a positive integer $m$ such that $S_{m}(n) \geqslant 0$ for all $n$.

Proof. Since $F(x, \chi)=\left(1-x^{k}\right)^{-1} f(x, \chi)$, (i) implies (ii). Since $f(x, \chi)$ is a polynomial, (ii) implies (iii) by Lemma 5 . Since

$$
\sum_{n=1}^{\infty} S_{m}(n) x^{n}=\left(1-x^{k}\right)^{-1} \sum_{n=1}^{\infty} s_{m}(n) x^{n}
$$

(iii) implies (iv). Since

$$
F(x, \chi)=(1-x)^{m} \quad \sum_{n=1}^{\infty} S_{m}(n) x^{n},
$$

(iv) implies (i).

LEMMA 7. If $\chi$ is a given nonprincipal residue character modulo $k$, let $m_{1}=$ $m_{1}(\chi)$ be the smallest positive integer such that $s_{m_{1}}(n) \geqslant 0$ for all $n$ and let $m_{2}=$ $m_{2}(\chi)$ be the smallest positive integer such that $S_{m_{2}}(n) \geqslant 0$ for all $n$. Then $m_{2} \leqslant$ $m_{1} \leqslant m_{2}+1$.

Proof. The first inequality follows from the above proof that (iii) implies (iv). The second follows from the identity 


$$
\sum_{n=1}^{\infty} s_{m+1}(n) x^{n}=\left(1+x+x^{2}+\cdots+x^{k-1}\right) \sum_{n=1}^{\infty} S_{m}(n) x^{n} .
$$

By the Fekete-Chowla method we mean the use of either (iii) or (iv) to test for the positivity of $f$ or $F$ throughout $(0,1)$. Properly speaking, Fekete considered the use of (iii) and in this he was followed by Grimm [3], while Chowla [1] discussed the use of (iv) and this was the procedure used by Rosser [9]. However, there is very little distinction between the two versions of the method.

In the case of the real nonprincipal characters modulo 1973 and 1277 the calculations discussed in the preceding section show that $f(x, \chi)$ comes close to having a real zero around 0.744 . In fact, in both cases $f$ has a pair of complex conjugate zeros in this vicinity, which we tried to approximate. We found that for the real nonprincipal character modulo 1973 the polynomial $f(x, \chi)$ has the following approximate quadratic factor

$$
x^{2}-1.488551225204145 x+0.5540322398114067
$$

while for the real nonprincipal character modulo 1277 the polynomial $f(x, \chi)$ has the following approximate quadratic factor

$$
x^{2}-1.489417749940728 x+0.5546548204910508 .
$$

(We do not claim that all the digits quoted here are significant.) If we use the inequality for $r$ quoted in the proof of Lemma 5 which guarantees that $(1-x)^{-r}\left(a x^{2}+b x+c\right)$ has positive coefficients, we find that the factor $(1-x)^{-568}$ will convert the quadratic factor for 1973 into a power series with positive coefficients and that the factor $(1-x)^{-766}$ will convert the quadratic factor for 1277 into a power series with positive coefficients.

We went through these efforts in order to get some idea of what power of $(1-x)^{-1}$ we must multiply by in order to convert $f(x, \chi)$ into a power series with positive coefficients. Actually the values obtained for the quadratic factors turned out to give a very good indication. For the real nonprincipal character modulo 1973 we found $m_{1}(x)=$ $m_{2}(x)=567$ instead of the suggested 568. For the real nonprincipal character modulo 1277 we found $m_{2}(\chi)=766$, exactly equal to the suggested value, but were unable to determine whether $m_{1}(\chi)$ is 766 or 767 without a further extensive calculation.

We proceed to give a discussion of how these values of $m_{2}(\chi)$ were obtained.

First let $\chi(n)=(n / 1973)$. Since $\chi(-1)=1$, we know that $S_{0}, S_{1}$, and $S_{2}$ have period 1973 and vanish at 1973. Calculation shows that $S_{3}(1973)=6096570$, so that $S_{3}(1973 m)=6096570 m$ for every positive integer $m$. The minimum value of $S_{2}(n)$ for $1 \leqslant n \leqslant 1973$ is -261 , attained at $n=157$ and 158 . Since $6096570+1973(-261)>0$, it follows that $S_{3}(n)>0$ for $n \geqslant 1973$. The computer found that $S_{3}(n) \leqslant 0$ for $6 \leqslant n \leqslant 17$ and for $156 \leqslant n \leqslant 429$ but that $S_{3}(n)>0$ for all other values of $n$ between 1 and 1973. Hence $S_{3}(n)>0$ for $n \geqslant 430$. 
Suppose now that $S_{m-1}(n)$ takes a block of nonpositive values when $n$ runs from $a$ to $b$ inclusive, where $1<a \leqslant b$. In other words suppose $S_{m-1}(a-1)>0, S_{m-1}(n) \leqslant 0$ for $a \leqslant n$ $\leqslant b$, and $S_{m-1}(b+1)>0$. Then either (1) $S_{m}(n)>0$ for $a \leqslant n \leqslant b$ (if $S_{m}(a-1)$ is very large), or (2) there is a $c$ such that $a \leqslant c \leqslant b, S_{m}(c-1)>0$, and $S_{m}(c) \leqslant 0$ (if $S_{m}(a-1)$ is positive but not very large), or (3) $S_{m}(n) \leqslant 0$ for $a-1 \leqslant n \leqslant b$ (if $S_{m}(a-1)$ $\leqslant 0$ ). Note that in cases (2) and (3) we must have $S_{m}(b) \leqslant 0$. Thus when we go from $S_{m-1}$ to $S_{m}$ a block of nonpositive values of $S_{m-1}$ can undergo one of the following transformations: (1) it can disappear, (2) its starting point can remain stationary or shift to the right but not go past the old ending point (while the length may change but must extend at least to the old ending point), (3) it can merge with a previous nonpositive block. Certainly a block of consecutive integers on which $S_{m-1}$ is nonpositive cannot split into two or more nonpositive blocks of $S_{m}$. In fact, these arguments show that the number of variations in sign among the values of $S_{m-1}$ can only decrease by an even number when we go over to $S_{m}$.

We know that $S_{3}$ has only two nonpositive blocks. For $1 \leqslant n \leqslant 1973$ computer calculations show that $S_{9}(n)$ is nonpositive only when $18 \leqslant n \leqslant 37$ or when $697 \leqslant n \leqslant 844$. Hence these blocks must have descended from the two nonpositive blocks of $S_{3}$. Since $S_{3}$ is always positive after its second nonpositive block, $S_{9}$ enjoys the same property, i.e., $S_{9}(n)>0$ for $n \geqslant 845$. In another calculation we found that $S_{10}(n) \leqslant 0$ for $22 \leqslant n \leqslant 42$ but $S_{10}(n)>0$ for all other positive integers $n \leqslant 1973$. Hence, while the second nonpositive block [156,429] of $S_{3}$ has descendants in $S_{4}, \cdots, S_{9}$, it does not have one in $S_{10}$, since $S_{10}(n)>0$ for $697 \leqslant n \leqslant 844$, so that no inheritance from the nonpositive block [697,844] in $S_{9}$ is possible. Hence $S_{10}(n)>0$ for $n \geqslant 43$.

The one remaining nonpositive block $[22,42]$ of $S_{10}$ has a descendant in each generation for some time. In fact a calculation determined that $S_{566}(1648)<$ $-10^{538}$ is the minimum value of $S_{566}$ on the interval $[1,1973]$ and that $S_{566}(1972)$ $>0$, so that $S_{566}$ has a single nonpositive block containing 1648 but not 1972. As a consequence $S_{566}(n)>0$ for all $n \geqslant 1972$. Finally we found that $S_{567}(n)>0$ for $1 \leqslant n \leqslant 1973$, so that the single nonpositive block appearing in $S_{10}$ through $S_{566}$ has finally disappeared. Thus $S_{567}(n)>0$ for all $n \geqslant 1$ and $m_{2}(\chi)=567$ in this case.

If we were to consider $s_{m}$ instead of $S_{m}$ in this argument for the nonprincipal real character modulo 1973, the only difference would be in the first paragraph of the argument. We would have $s_{0}(n)=0$ for $n \geqslant 1973, s_{1}(n)=0$ for $n \geqslant 1973$, $s_{2}(n)=0$ for $n \geqslant 1973$, and $s_{3}(n)=6096570$ for $n \geqslant 1973$. But the nonpositive blocks of $S_{3}, \cdots, S_{566}$ all terminate before 1973 and so would be exactly the same for $s_{3}, \cdots, s_{566}$. Thus $m_{1}(\chi)=567$ in this case.

Now we discuss the corresponding calculations for the real nonprincipal character modulo 1277. As before $S_{0}, S_{1}$, and $S_{2}$ have period 1277 and vanish at 1277 . We found $S_{3}(1277)=2045754$ and so $S_{3}(1277 \mathrm{~m})=2045754 \mathrm{~m}$ for every positive 
integer $m$. The minimum value of $S_{2}(n)$ for $1 \leqslant n \leqslant 1277$ is -149 , attained for 8 values less than 1277 . Since $2045754+1277(-149)>0$, we infer that $S_{3}(n)>0$ for $n \geqslant 1277$. The computer found that $S_{3}(n) \leqslant 0$ for $6 \leqslant n \leqslant 17$ and for $96 \leqslant$ $n \leqslant 282$ and that $S_{3}(n)>0$ for all other values of $n$ less than 1277. Accordingly $S_{3}(n)>0$ for $n \geqslant 283$.

Just as for the real nonprincipal character modulo 1973, we know that $S_{m}$ for $m \geqslant 3$ can have at most two nonpositive blocks. Two nonpositive blocks do exist for $S_{23}$, since $S_{23}(n) \leqslant 0$ for $54 \leqslant n \leqslant 85$ and for $1167 \leqslant n \leqslant 1236$ but $S_{23}$ is otherwise positive on [1, 1277]. However, the second nonpositive block disappears when we go from $S_{23}$ to $S_{24}$, since $S_{24}(n) \leqslant 0$ for $57 \leqslant n \leqslant 88$ but $S_{24}$ is otherwise positive on $[1,1277]$. Thus $S_{24}(n)>0$ for $n \geqslant 89$ and $S_{m}$ has at most one nonpositive block for any $m$ greater than 23 .

A calculation determined that $S_{765}(2232)<0$ while $S_{765}(2500)>0$. Thus $S_{765}$ has a nonpositive block which includes 2232 but not 2500 , and this is its only nonpositive block. Finally we found that $S_{766}(n)>0$ for $1 \leqslant n \leqslant 2500$. If there were a nonpositive block in $S_{766}$, it would have to begin before 2500 . Thus $S_{766}(n)$ $>0$ for all positive integral $n$ in the case of the real nonprincipal character modulo 1277. Note that the nonpositive block of $S_{765}$ extends well beyond 1277 , which complicates the precise determination of $m_{1}(\chi)$. But $766=m_{2}(\chi) \leqslant m_{1}(\chi) \leqslant 767$.

7. Concluding Remarks. In [4] Hahn has pointed out that a slight modification of the arguments in [5] gives the following generalization of Heilbronn's theorem quoted in Section 1. Let $p_{i}$ denote the $i$ th prime. Suppose $\epsilon_{1}, \cdots, \epsilon_{h}$ are given, where each $\epsilon_{i}$ is $-1,0$, or 1 . Then there is a positive integer $r$ with the following property. For any real residue character $\chi$ such that

$$
\chi\left(p_{i}\right)=\epsilon_{i} \text { for } i=1,2, \cdots, h,
$$

and

$$
\chi\left(p_{i}\right)=-1 \text { for } i=h+1, \cdots, h+r
$$

the corresponding Fekete polynomial $f(x, \chi)$ changes sign in $(0,1)$.

In this paper we showed that when $h=0$ the smallest value of $r$ which would work is $r=5$. In principle it would be possible to determine the smallest admissible value of $r$ in other cases as well.

Hahn's generalization of Heilbronn's theorem heuristically indicates that if $\chi$ is a real primitive character to a very large modulus, then $f(x, \chi)$ is very likely to have a sign change in $(0,1)$. For at any stage a sufficiently long string of minus ones among the values of $\chi(p)$ is enough to force the existence of such a sign change. If the modulus is large enough, there are plenty of such opportunities to go astray. To put it another way, assertions I, II, and III show that prescribing the values of $\chi(p)$ for a finite set of primes $p$ can guarantee the existence of a sign change, but Hahn's general- 
ization of Heilbronn's theorem shows that no such prescription can guarantee positivity throughout $(0,1)$. Thus it is reasonable to conjecture that in some sense most real primitive characters have the property that their Fekete polynomials change signs in $(0,1)$. A rigorous proof of some such result would be of great interest.

Department of Mathematics

University of Illinois at Urbana-Champaign

Urbana, Illinois 61801

1. S. CHOWLA, "Note on Dirichlet's $L$-functions," Acta Arith., v. 1, 1935, pp. 113-114.

2. M. FEKETE \& G. PÓlyA, “Über ein Problem von Laguerre," Rend. Circ. Mat. Palermo, v. 34, 1912 , pp. 89-120.

3. GOTTFRIED GRIMM, Über die reellen Nullstellen Dirichlet'scher L-Reihen, Dissertation, E. T. H., Zurich, 1932.

4. HWA S. HAHN, “On a conjecture of Fekete," J. Korean Math. Soc., v. 5, 1968, pp. $13-16$.

5. H. HEILBRONN, “On real characters," Acta Arith., v. 2, 1937, pp. 212-213.

6. M. E. LOW, "Real zeros of the Dedekind zeta function of an imaginary quadratic field," Acta Arith., v. 14,1967/68, pp. 117-140. MR 38 \#4425.

7. GEORG PÓLYA, “Verschiedene Bemerkungen zur Zahlentheorie," Jber. Deutsch. Math. Verein., v. 28, 1919, pp. 31-40.

8. G. PÓLYA \& G. SZEGÖ, Aufgaben und Lehrsätze aus der A nalysis. Vol. 2, Springer, Berlin, 1925.

9. J. BARKLEY ROSSER, "Real roots of real Dirichlet L-series," J. Res. Nat. Bur. Standards, v. 45, 1950, pp. 505-514. MR 12, 804.

10. J. H. WILKINSON, Rounding Errors in Algebraic Processes, Prentice-Hall, Englewood Cliffs, N. J., $1963 . \quad$ MR 28 \#4661. 